\title{
Dual-Curing, Self-Adhesive Resin Cement: Influence of the Polymerization Modes on the Degree of Conversion and Microhardness
}

\author{
Luis Claudio Mendes ${ }^{\mathrm{a} *}$, Irma Cunha Matos ${ }^{\mathrm{b}}$, Mauro Sayão Miranda ${ }^{\mathrm{b}}$, Marcia Regina Benzi ${ }^{\mathrm{a}}$ \\ ${ }^{a}$ Centro de Tecnologia, Instituto de Macromoléculas Professora Eloisa Masno - IMA, \\ Universidade Federal do Rio de Janeiro - UFRJ, Bloco J, PO Box 68525, \\ Av. Horácio Macedo, 2030, CEP 21941-598, Rio de Janeiro, RJ, Brazil \\ ${ }^{\mathrm{b}}$ Departamento de Dentística, Escola de Dentística, \\ Universidade do Estado do Rio de Janeiro - UERJ, Rio de Janeiro, RJ, Brazil
}

Received: October 30, 2009; Revised: March 15, 2010

\begin{abstract}
Rely $\mathrm{X}^{\mathrm{TM}}$ Unicem, dual-curing self-adhesive resin cement, was tested in order to evaluate the degree of conversion (DC) and Vickers microhardness (VMH). The specimens were prepared into 4 groups (GR). For GR1, the specimens were irradiated with $500 \mathrm{~mW} \cdot \mathrm{cm}^{-2}$ during 20 seconds while for GR3 the same intensity was used but the irradiation time was 40 seconds. The GR2 followed the same polymerization mode of the GR1 but a ceramic disk was applied as a light barrier. For the last group, only the chemical polymerization was carried out. The DC and VMH were measured immediately after the irradiation time and again after 24 hours. Dual-polymerization (light and chemical polymerization) showed higher DC and VMH than the chemical polymerization (dark cure). The presence of a ceramic disk did not affect the DC but reduced the VMH. Increasing the exposure to light, the DC remained almost constant whereas the VMH raised slightly indicating that the VMH is quite dependent on the incident energy. Additionally, the powder/liquid portions of the cement were also characterized. The TG showed that the solid fraction was constituted of $96 \%$ of inorganic matter and $4 \%$ of vinyl-silane, a coupling agent. Regarding the liquid part, it was found that $84 \%$ was related to the mixture of monomers. The SEM revealed that the size particle of the inorganic fillers were between 400 and $11.000 \mathrm{~nm}$. Factors such as energy density, size and distribution of inorganic fillers and the low effectiveness of the chemical cure contributed to the low values of the DC and VMH.
\end{abstract}

Keywords: dual-curing cement, self-adhesive cement, degree of conversion, vickers microhardness

\section{Introduction}

The use of restoration cements has been growing jointly with the increase of ceramics and composite resins for repairing teeth. Although these materials show high mechanical strength and remarkable esthetic appeal, the filling of the tooth cavity requires multiple steps and consumes a lot of time ${ }^{1}$. Self-adhesive resin cements have been developed to eliminate pre-treatment steps such as etching, priming and bonding when compared to multi-step composite cements. However, they need clinical and in vitro studies in the long-term, dimensional stability, their risks and benefits and so on. An important aspect to be considered is the monomer's degree of conversion which affects the physico-chemical properties including strength, stiffness, water absorption, biocompatibility as well as color stability. Post-operative sensitivity has also been reported as a possible consequence of inadequate polymerization of the resin cement ${ }^{2-13}$. The use of dual-curing resin cements intends to combine chemical and light-polymerization and at the same time allowing polymerization to take place in deeper areas of the tooth cavity applying shorter light irradiation time. Several studies reported that when photoactivation is omitted i.e. dark-curing took place and the material was unable to attain a high degree of monomer conversion ${ }^{14-24}$. Indeed, the degree of polymerization is still influenced when a light barrier simulating a porcelain indirect restoration is placed between the light source and

*e-mail: lcmendes@ima.ufrj.br the cement. Several studies showed adverse effects on the degree of conversion and microhardness of the dual-curing and lightpolymerized resin cements with the increase of the thickness of the light barrier material ${ }^{25-31}$.

The purpose of this study was to evaluate the degree of conversion and microhardness of one commercial dual-curing self-adhesive resin cement, named RelyX $\mathrm{X}^{\mathrm{TM}}$, polymerized using different protocols, considering the polymerization mode, irradiation time and the use of a ceramic disk as a light barrier. Additionally, the powder/ liquid portions of the cement were characterized in order to help the evaluation of the cement's behavior with regard to the polymerization conditions.

\section{Experimental}

\subsection{Material and sample preparation}

Cylindrical specimens $(0.5 \mathrm{~mm}$ height and $8.0 \mathrm{~mm}$ diameter $)$ were prepared in a Teflon ${ }^{\circledR}$ mold with RelyX ${ }^{\mathrm{TM}}$ Unicem Aplicap (shade A2, 3M ${ }^{\circledR}$ ESPE Dental Products, St Paul, MN, USA). The cement constituted two portions - one liquid and the other solid. The specimens were separated into 4 groups according to different preparation protocols. The degree of conversion and the Vickers 
microhardness were measured immediately after the irradiation time. After that, the specimens were kept in the light-proof box, at $37^{\circ} \mathrm{C}$, and after 24 hours those properties were also evaluated.

A ceramic disk similar to the microscopic glass slide $(2.0 \mathrm{~mm}$ height and $10.0 \mathrm{~mm}$ diameter) was molded using IPS Empress 2 (shade A2, Ivoclar ${ }^{\circledR}$ Vivadent, Schan, Liechtenstein) following the manufacturer's instructions in order to be used as a light barrier simulating a porcelain indirect restoration.

For Group 1 (GR1), the specimen preparation followed strictly the manufacturer's instructions by placing the resin cement into the mold, covered with a Mylar strip and a microscope glass slide. After that, the cement was polymerized using a light curing unit (Optilux 400, Demetron, Kerr Corp, Orange, CA,USA) with $500 \mathrm{~mW} \cdot \mathrm{cm}^{-2}$ of light intensity, with an irradiation time of 20 seconds.

The specimens of Group 2 (GR2) were polymerized under the same conditions but the microscope glass slide was replaced by a ceramic disk in order to simulate a porcelain indirect restoration. The specimens of Group 3 (GR3) were obtained under the same conditions as those of Group 2 but the irradiation time was higher (40 seconds) with the purpose of assessing its influence on the degree of polymerization.

For Group 4 (GR4), the cement was inserted into the mold and then put into a light-proof box, kept for five minutes, then for 24 hours in order to ensure only chemical/dark curing took place, as the maker had suggested. For both reaction times, the degree of conversion and microhardness were measured.

\subsection{Fourier transform infrared spectroscopy (FT-IR)}

The degree of conversion was measured using Varian equipment model Excalibur 3100 equipped with an attenuated total reflectance assembly, with 100 scans and $4 \mathrm{~cm}^{-1}$ of resolution. The calculation of the degree of conversion was based on the ratio between the absorbances at 1637 and $1610 \mathrm{~cm}^{-1}$, according to the previous work ${ }^{32}$. The results represented the average of five measurements.

\subsection{Vickers microhardness (VHM)}

Hardness is a general term which describes a combination of properties, such as the resistance to penetration, abrasion and scratching. It is an important parameter concerning the mastication and teeth' cleaning and they are the reasons for it large application in physical characterization of dental restoration. The Vickers microhardness test was carried out with a Carl Zeiss ${ }^{\circledR}$ Neophot 2 microdurometer, with load of $0.1 \mathrm{Kgf}$ for 30 seconds. The results represented the average of five measurements.

\subsection{Thermogravimetry (TG)}

The thermogravimetry was carried out in a TA thermogravimetric analyzer model Q500 in the range of $30-700{ }^{\circ} \mathrm{C}$, at $10^{\circ} \mathrm{C} / \mathrm{min}$, under nitrogen atmosphere.

\section{5. ${ }^{13} \mathrm{C}$ and ${ }^{31} \mathrm{P}$ nuclear magnetic resonance (NMR)}

The nuclear magnetic resonance of carbon-13 $\left({ }^{13} \mathrm{C} \mathrm{NMR}\right)$ of the solid and liquid portions of the cement was performed in Varian equipment model Mercury 300, at a frequency of 75.4 MHz. The portions were dissolved in deutered chloroform, analyzed at $25{ }^{\circ} \mathrm{C}$, in order to estimate the chemical composition of the cement ${ }^{32}$.

The nuclear magnetic resonance of phosphorus $31\left({ }^{31} \mathrm{P}\right.$ NMR) was also used to confirm the presence of combined phosphorus in the liquid portion of the cement.

\subsection{Scanning electron microscopy (SEM)}

The scanning electron microscopy was performed using JEOL ${ }^{\circledR}$ equipment, model JSM 5600, in order to observe the size and distribution of inorganic filler in the cement. A post-polymerized fragment was metallized with gold from which a photograph was taken.

\section{Results and Discussion}

\subsection{Fourier transform infrared spectroscopy (FT-IR)}

The solid portion of the cement indicated infrared absorptions at $1100-1000$ and $800 \mathrm{~cm}^{-1}$ which were attributed to Si-O-C, $\mathrm{S}$ i-O-Si and $\mathrm{Si}-\mathrm{OH}$ linkages. These absorptions are related to the presence of silica as one of the fillers and possibly vinyl-silane, used as a coating of the fillers in this portion, in view of the increase in the interfacial adhesion between the organic and inorganic phases. Relating to the liquid phase, the infrared absorptions were noticed at $1716,1637,1620,1582$ and $1510 \mathrm{~cm}^{-1}$ and in the interval between $1300-1000$ and $900-650 \mathrm{~cm}^{-1}$. These are related to the symetric and assymetric stretching of the ester carbonyl, double linkage of the vinyl monomer and stretching and bending of the $\mathrm{C}=\mathrm{C}$ and $\mathrm{C}-\mathrm{H}$ links of the aromatic ring. Thus, the liquid phase seems to contain monomers of the unsaturated ester type, and considering the absorptions with reference to the aromatic ring, one may conclude that the monomer Bisphenol-A diglycidylether methacrylate-Bis-GMA, usually employed as the main vinylic monomer in the formulation of dental restoration composites, is one of the monomers employed in the formulation of the cement ${ }^{33-35}$.

\section{2. ${ }^{13} \mathrm{C}$ and ${ }^{31} \mathrm{P}$ nuclear magnetic resonance (NMR)}

In Figures 1 and 2, respectively present the nuclear magnetic resonance spectra of carbon-13 of the solid and liquid portions of the cement. In Table 1 are listed the main chemical displacements. In relation to the solid fraction, the chemical displacements could be attributed to the unsaturated carbon-carbon linkage and would correspond to vinyl-silane, confirming the FT-IR results. With reference to the liquid phase, the ${ }^{13} \mathrm{C}$ NMR revealed inumerous chemical displacementes. Comparing it with spectra of known samples of monomers usually employed in dental composite formulae, it was verified that the displacements - 18.0; 30.9; 41.5; $61.9 ; 65.3 ; 113.6 ; 126.2 ; 126.4 ; 127.5 ; 136.0 ; 156.4 ; 166.3$ and $167.2 \mathrm{ppm}$ - would be related to the monomer Bis-GMA while those at $18.0 ; 63.7 ; 125.6 ; 136.0$ and $166.8 \mathrm{ppm}$ characterize the presence of the monomer triethyleneglycol dimethacrylate - TEGDMA. The relative percentual of each monomer was calculated considering the peak areas of chemical displacements at $65.3 \mathrm{ppm}$ (Bis-GMA) and $63.7 \mathrm{ppm}$ (TEGDMA), according to the previously described procedure $^{32}$. Thus, the monomeric fraction of the liquid fraction of the cement is $18.6 \%$ of Bis-GMA and $81.4 \%$ of TEGDMA. The higher amount of TEGDMA, almost 4 times higher than Bis-GMA, plays a role as diluent owing to its low viscosity ${ }^{33-34}$. Thus, a greater quantity of diluent in the monomeric composition aimed to facilitate the diffusion of monomers in the reactional medium and permit their penetration in deeper areas of the tooth cavity.

Further, in the liquid phase a nuclear magnetic resonance analysis of phosphorus-31 was performed, in an attempt to characterize the presence of organic phosphate in the cement (Figure 3). The analysis showed a broad peak where the maximum displacement occurred at $1.727 \mathrm{ppm}$, which could be attributed to the presence of a phosphorusester of the methacrylate monomer, which the finality would be to promote anchorage of the vinylic monomer to the tooth surface. 


Dentis \& PO
LAPSER
Carbono 13 Quanti.
RMN 436-07
Oper.: E.Miguez

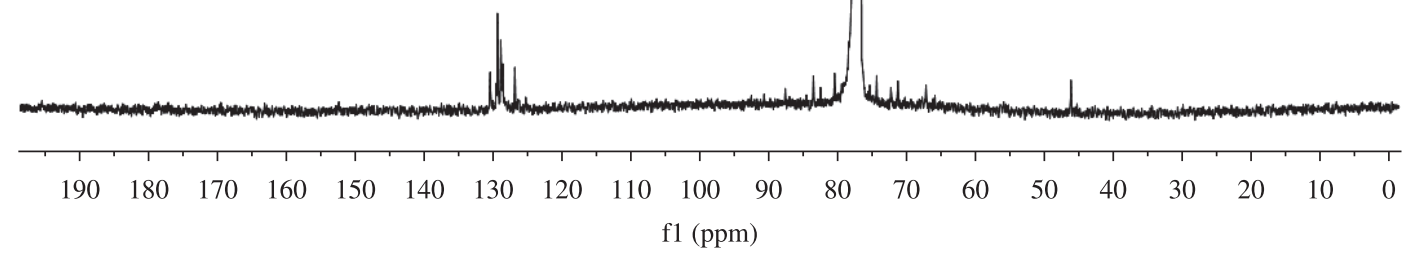

Figure 1. ${ }^{13} \mathrm{C}$ NMR spectrum of the cement solid portion.

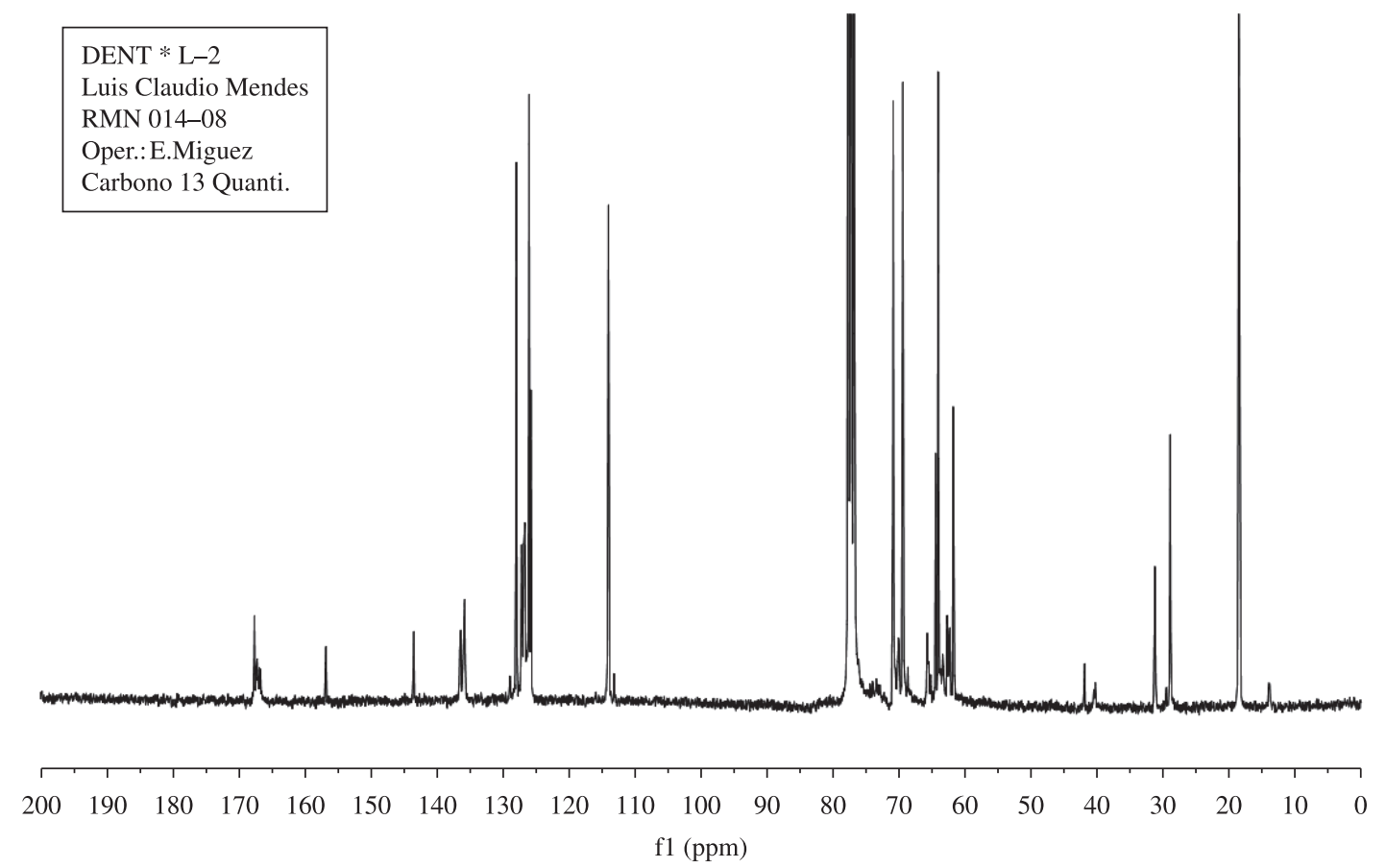

Figure $2 .{ }^{13} \mathrm{C}$ NMR spectrum of the cement liquid portion.

\subsection{Thermogravimetry (TG)}

Thermogravimetry indicated that the solid fraction constituted $96 \%$ of the inorganic volume and $4 \%$ of the organic material which was related to vinyl-silane, used as a coating with the view of improving the interfacial adhesion of the composite. Regarding the liquid fraction, the mass loss was $84 \%$ further revealing an incombustable portion of $16 \%$ which could be related to the phosphorus pentoxide residue and incomplete burning of organic material due to the nonoxidizing nitrogen atmosphere.
Table 1. ${ }^{13} \mathrm{C}$ NMR chemical displacements of the cement solid and liquid portions.

\begin{tabular}{cc}
\hline Portion & $\begin{array}{c}\text { Chemical displacement } \\
(\mathrm{ppm})\end{array}$ \\
\hline Solid & $46.1 ; 126-130$ \\
Liquid & $13.6 ; 18.0 ; 28.5 ; 30.9 ; 39.9 ; 41.5 ; 61.4 ; 61.9 ; 62.1 ; 62.3 ;$ \\
& $63.0 ; 63.7 ; 64.1 ; 64.8 ; 65.3 ; 68.2 ; 69.0 ; 69.7 ; 112.7 ; 113.6 ;$ \\
& $125.3 ; 125.6 ; 126.2 ; 126.4 ; 126.8 ; 127.5 ; 128.5 ; 135.4 ;$ \\
& $136.0 ; 136.1 ; 143.1 ; 156.4 ; 166.3 ; 166.5 ; 166.8 ; 167.2$ \\
\hline
\end{tabular}




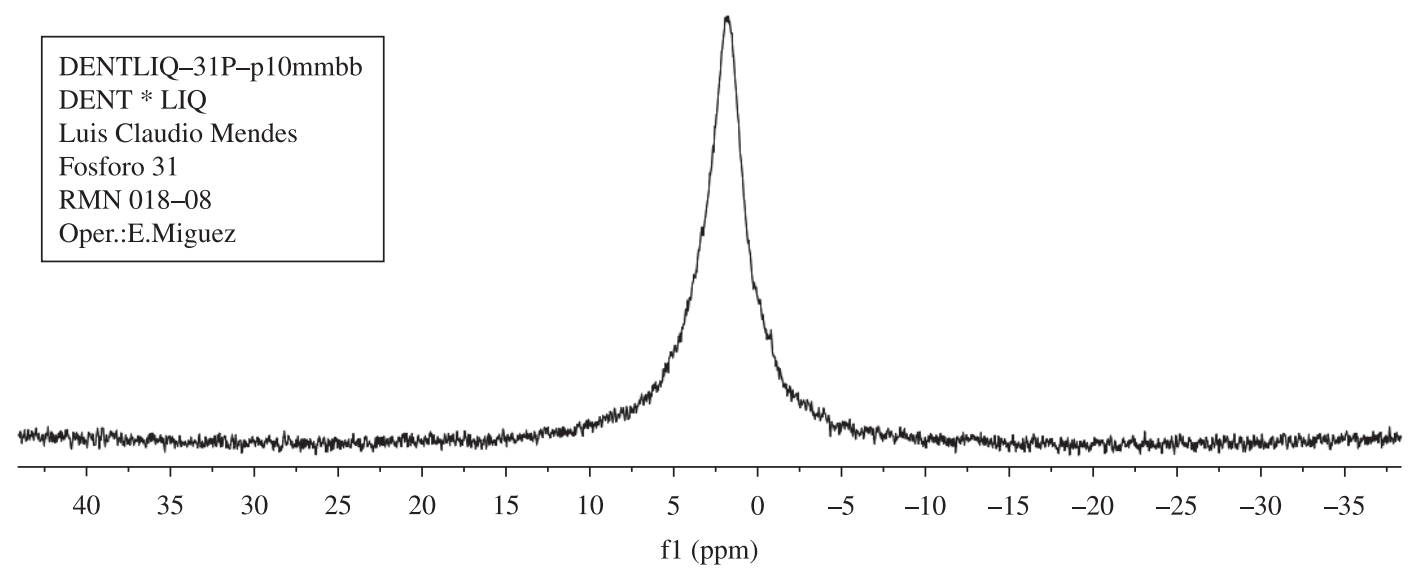

Figure 3. ${ }^{31} \mathrm{P}$ NMR spectrum of the cement liquid portion.

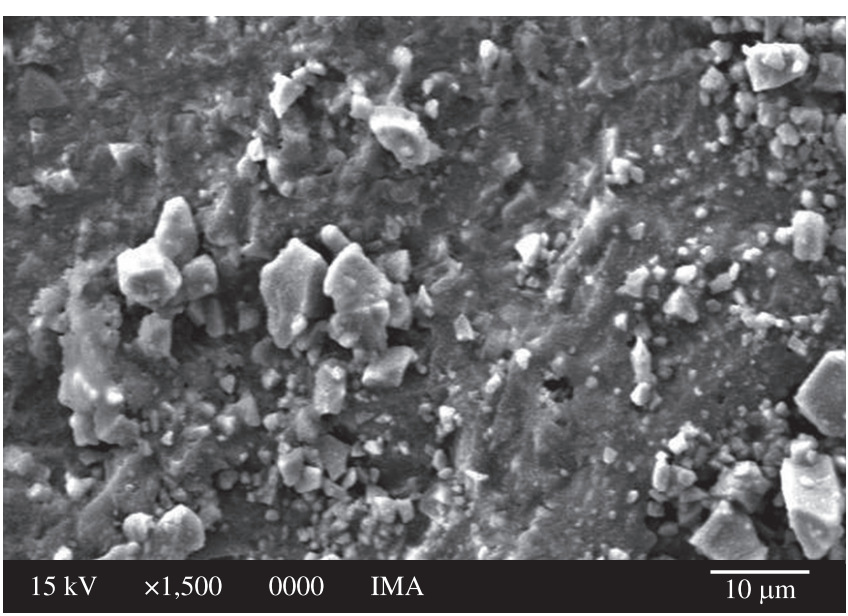

Figure 4. SEM photomicrograph of the polymerized composite.

\subsection{Scanning electron microscopy (SEM)}

A photomicrograph of the test specimen, after polymerization (Figure 4), indicated there is a great variation of size and form of the particles of the inorganic portion. Particle size varied between $0.4-11.2$ microns $(400-11.200 \mathrm{~nm})$.

\subsection{Degree of conversion (DC)}

In Table 2 are listed the degree of conversion for the different groups. The specimens polymerized in the presence of light showed values of DC almost 4 times superior to that of the group whose polymerization occurred in the absence of light. After 24 hours, it was verified that the test specimens polymerized by light had an increase in the degree of conversion of around $10 \%$ while for those polymerized chemically an increase of around $150 \%$ was observed. Although being a considerable percentage, the DC value was lower ( $60 \%)$ to those observed for GR1, GR2 and GR3. Regarding the presence of the ceramic disk and the increase in light exposure time on the cement, it appears these did not influence the degree of conversion.

\subsection{Vickers microhardness}

In Table 3 are listed the Vickers microhardness values of the different groups. It was observed that this property was greatly dependent on the polymerization conditions. With respect to the test specimens of groups GR1, GR2 and GR3, the VMH values varied due to the ceramic disk and with light exposure time. After 24 hours,
Table 2. Degree of conversion of the different specimen groups.

\begin{tabular}{cccc}
\hline Group & \multicolumn{3}{c}{ Degree of conversion (\%) } \\
\cline { 2 - 4 } & $\begin{array}{c}\text { Polymerization } \\
\text { mode }\end{array}$ & $\begin{array}{c}\text { Immediately after } \\
\text { light exposition }\end{array}$ & $\begin{array}{c}\text { 24 hours after } \\
\text { light exposition }\end{array}$ \\
\hline GR1 & light-curing & $29.6 \pm 4.0$ & $32.6 \pm 3.0$ \\
GR2 & light-curing & $27.1 \pm 2.4$ & $28.9 \pm 3.9$ \\
GR3 & light-curing & $28.2 \pm 3.6$ & $32.3 \pm 4.0$ \\
- & - & After 5 minutes & After 24 hours \\
GR4 & dark-curing & $7.9 \pm 2.4$ & $20.4 \pm 4.9$ \\
\hline
\end{tabular}

Table 3. Vickers microhardness of the different specimen groups.

\begin{tabular}{cccc}
\hline Group & \multicolumn{3}{c}{ Vickers microhardness } \\
\cline { 2 - 4 } & $\begin{array}{c}\text { Polymerization } \\
\text { mode }\end{array}$ & $\begin{array}{c}\text { Immediately after } \\
\text { light exposition }\end{array}$ & $\begin{array}{c}\text { 24 hours after } \\
\text { light exposition }\end{array}$ \\
\hline GR1 & light-curing & $8.0 \pm 1.8$ & $14.9 \pm 2.3$ \\
GR2 & light-curing & $4.8 \pm 0.1$ & $9.1 \pm 1.6$ \\
GR3 & light-curing & $6.5 \pm 2.5$ & $11.4 \pm 2.0$ \\
- & - & After 5 minutes & After 24 hours \\
GR4 & dark-curing & - & $10.7 \pm 0.9$ \\
\hline
\end{tabular}

the VMH values were around twice as high to those determined at the end of light irradiation. It was not possible to measure the VMH of group GR4 after five minutes reaction time because the composite was not sufficiently rigid. Although the test specimens of GR4 resulted in an average value of DC inferior to those activated by light, the average of microhardness was in the same order of magnitude after 24 hours of reaction.

The presence of a ceramic disk influenced the microhardness of the composite. Lesser values of this property were found for groups GR2 and GR3 in relation to GR1 (glass slide). The average VMH value of group GR3 was sharply superior to group GR2, indicating that increase of light exposure time also affected this property. Although it did not alter the DC value, the presence of the ceramic disk significantly reduced the $\mathrm{VMH}$, indicating that this property is very dependent on the quantity of incident light in the polymerization system. Thus, microhardness is related as much to the DC as to the degree of crosslinking.

With the light irradiation, the initiation step occurs instantaneously due to the production of free radicals. The propagation step proceeds 
and there is an increase of the viscosity of the composite making difficult the monomer diffusion to the propagation centers of the polymeric chain. This is one of the reasons that could be attributed to the low values of the DC and VHM. Further factors such as energy density (light intensity $\times$ exposition time), size and distribution of the inorganic fillers could be also considered. Braga, Ozyesil, Santos Jr. and Rasetto ${ }^{7-8,17,20}$ reported that the increase of the energy density led to the increase of the DC in their studies on dual resin cements. In fact, Kumbuloglu et al. ${ }^{16}$ mentioned that $56 \%$ of DC was achieved when $32 \mathrm{~J} . \mathrm{cm}^{-2}$ of the energy density was applied to dual-curing cement. The energy density irradiated to the RelyX ${ }^{\mathrm{TM}}$ was 10 and $20 \mathrm{~J} . \mathrm{cm}^{-2}$. It is 2-3 times lower than that used by Kumbuloglu and then, it would be not enough to attain higher values of DC and VMH. The size and distribution of the inorganic fillers could be also mentioned. The SEM analysis revealed that the size particles were between 400 and $11.000 \mathrm{~nm}$. Considering that the wavelength of the incident light used in this work was $470 \mathrm{~nm}$, there was a great scattering of the light and its transmission to the liquid portion was unable to lead to higher values of degree of conversion and crosslinking, both straight related to the microhardness.

There is not influence of the ceramic disk which simulated a porcelain indirect restoration on the properties measured herein. The result is in agreement with Prinsloo' ${ }^{25}$ study. He mentioned that only disk with thickness upper than $2 \mathrm{~mm}$ modified significantly the DC.

The chemical curing (without light), in both polymerization modes, seems to have low effectiveness. Its activation was extremely slow making the chemical polymerization system a little inefficient and in the presence of light its contribution on the composite properties would be neglected.

\section{Conclusion}

Dual-polymerization mode showed higher DC and VMH than the chemical polymerization one. The ceramic disk did not affect the DC but reduced the VMH. The VHM seems to be more dependent on the incident energy than the DC. The energy density, size and distribution of the inorganic fillers and the low effectiveness of the chemical initiation led to the low values of the monomeric conversion and microhardness.

\section{Acknowledgements}

The authors thank Conselho Nacional de Desenvolvimento Científico e Tecnológico (CNPq), Fundação Coordenação do Aperfeiçoamento de Pessoal de Nível Superior (CAPES), Universidade Federal do Rio de Janeiro (UFRJ) and Universidade do Estado do Rio de Janeiro (UERJ) for supporting this investigation.

\section{References}

1. Rosenstiel SF, Land MF and Crispin BJ. Dental luting agents: A review of the current literature. The Journal of Prosthetic Dentistry. (Saint Louis) 1998; 80(3):280-301.

2. El-Mowafy OM and Rubo MH. Influence of composite inlay/onlay thickness on hardening of dual-cured resin cements. Journal of the Canadian Dental Association. (Toronto) 2000; 66(3):147.

3. El-Mowafy OM, Rubo MH and El-Badrawy WA. Hardening of new resin cements cured through a ceramic inlay. Operative Dentistry. (Washington, DC) $1999 ; 24(1): 38-44$.

4. Ferracane JL. Correlation between hardness and degree of conversion during the setting reaction of unfilled dental restorative resins. Dental Materials (Kidlinton) 1985; 1(1):11-14, 1985.

5. Meng X, Yoshida K and Atsuta M. Influence of ceramic thickness on mechanical properties and polymer structure of dual-cured resin luting agents. Dental Materials. (Kidlinton) 2008; 24(5):594-599.
6. Asmussen E. Restorative resins: hardness and strength vs. quantity of remaining double bonds. Scandinavian Journal of Dental Research. (Washington) 1982; 90(6):484-489.

7. Braga RR and Ferracane JL. Contraction stress related to degree of conversion and reaction kinetics. Journal of Dental Reasearch. (Washington) 2002; 81(2):114-118.

8. Rasetto F, Driscoll CF and von Fraunhofer JA. Effect of light source and time on the polymerization of resin cement through ceramic veneers. Journal of Prosthodontics: Implant, Esthetic and Reconstructive Dentistry. (Philadelphia) 2001; 10(3):133-139.

9. Akgungor G, Akkayan B and Gaucher, H. Influence of ceramic thickmess and polymerization mode of a resin luting agent on early bond strength and durability with a lithium disilicatee-based ceramic system. The Journal of Prothetic Dentistry (Saint Louis) 2005; 94(3):234-241.

10. Ceballos L, Garrido MA, Fuentes V and Rodríguez J. Mechanical characterization of resin cements used for luting fiber posts by nanoindentation. Dental Materials. (Kidlinton) 2007; 23(12):100-105.

11. Hofmann N, Papsthart G, Hugo B and Klaiber B. Comparison of photoactivation versus chemical or dual-curing of resin-based luting cements regarding flexural strength, modulus and surface hardness. Journal of Oral Rehabilitation. (Oxford) 2001; 28(11):1022-1028.

12. Lu H, Mehmood A, Chow A and Powers JM. Influence of polymerization mode on flexural properties of esthetic resin luting agents. The Journal of Prothetic Dentistry. (Saint Louis) 2005; 94(6):549-554.

13. Radovic I, Monticelli F, Goracci C, Vulicevic ZR and Ferrari M. Adhesive resin cements: a literature review. Journal of Adhesive Dentistry. (Carol Stream) 2008; 10(4):251-258.

14. Braga RR, Cesar PF and Gonzaga CC. Mechanical properties of resin cements with different activation modes. Journal of Oral Rehabilitation. (Oxford) 2002; 29(3):257-262.

15. Jung H, Friedl KH, Hiller KA, Haller A and Schmalz G. Curing efficiency of different polymerization methods through ceramic restorations. Clinical Oral Investigations. (Berlin) 2001; 5(3):156-161.

16. Kumbuloglu O, Lassila LV, User A and Vallittu PK. A study of the physical and chemical properties of four resin composite luting cements. The International Journal of Prosthodontics. (Lombard) 2004; 17(3):357-363

17. Ozyesil AG, Usumez A and Gunduz B. The efficiency of different light souces to polymerize composite beneath a simulated ceramic restoration. The Journal of Prothetic Dentistry. (Saint Louis) 2004; 91(2):151-157.

18. Papazoglou E, Rahiotis C, Kakaboura A and Loukidis M. Curing efficiency of a photo- and dual-cured resin cement polymerized through 2 ceramics and a resin composite. The International Journal of Prosthodontics. (Lombard) 2006; 19(1):34-36.

19. Rueggeberg FA and Caughman WF. The influence of light exposure on polymerization of dual-cure resin cements. Operative Dentistry. (Washington, DC) 1993; 18(2):48-55.

20. Santos Jr GC, El-Mowafy O, Rubo JH and Santos MJ. Hardening of dual-cure resin cements and a resin composite restorative with QTH and LED light curing. Journal of Canadian Dental Association. (Toronto) 2004; 70(5):323-328.

21. Arrais CA, Giannini M and Rueggeberg FA. Kinetic analysis of monomer conversion in auto- and dual-polymerizing modes of commercial resin luting cements. Journal of Prosthetic Dentistry. (Saint Louis) 2009; 101(2):128-136, 2009.

22. Moraes RR, Brandt WC, Naves LZ, Correr-Sobrinho L and Piva E. Light- and time-dependent polymerization of dual-cured resin luting agent beneath ceramic. Acta Odontológica Scandinavica. (Oslo) 2008; 66(5):257-261.

23. Arrais CA, Rueggeberg FA, Waller JL, Goes MF and Giannini M. Effect of curing mode on the polymerization characteristics of dual-cured resin cement systems. Journal of Dentistry. 2008; 36(6):418-426.

24. Pazin MC, Moraes RR, Gonçalves LS, Borges GA, Sinhoreti MA, Correr-Sobrinho L. Effects of ceramic thickness and curing unit on light transmission through leucite-reinforced material and polymerization 
of dual-cured luting agent. Journal of Oral Science. (Tokyo) 2008; 50(2):131-136.

25. Prinsloo LC, van der Vyver PJ, Ferreira MR and Heyns AM. Percentage cure of cement cured through various thicknesses of Cerec porcelain. Journal of the Dental Association of South Africa. (Pretória) 1997; 52(5):283-286.

26. Tango RN, Sinhoreti MA, Correr AB, Correr-Sobrinho L and Henriques GE. Effect of Light-Curing Method and Cement Activation Mode on Resin Cement Knoop Hardness. Journal of Prosthodontics (Philadelphia) 2007; 16(6):480-484.

27. Yoshida K, Tsuo Y, Meng X and Atsuta M. Mechanical Properties of Dual-Cured Resin Luting Agents for Ceramic Restoration. Journal of Prosthodontics. (Philadelphia) 2007; 16(5):370-376.

28. Peixoto RT, Paulinelli VM, Sander HH, Lanza MD, Cury LA and Poletto LT. Light transmission through porcelain. Dental Materials. (Kidlinton) 2007; 23(11):1363-1368.

29. Meng X, Yoshida K and Atsuta M. Hardness Development of Dual-cured Resin Cements through Different Thicknesses of Ceramics. Dental Materials (Kidlinton) 2006; 25(1):132-137.

30. Vieno S, Madini L, Barabanti N, Alessandri I, Gagliani M and Cerutti A. Indirect resin composite restorations: evaluation of polymerization of luting agents by means of micro-Raman spectrophotometry. Minerva Stomatologica (Turin) 2009; 58(1-2):1-8.

31. Ilie $\mathrm{N}$ and Hickel R. Correlation between ceramics translucency and polymerization efficiency through ceramics. Dental Materials. (Kidlinton) 2008; 24(7):908-914.

32. Mendes LC, Tedesco AD and Miranda MS. Determination of degree of conversion as function of depth of a photo-initiated dental restoration composite. Polymer Testing (Essex) 2005; 24(4):418-422.

33. Mendes LC, Tedesco AD, Miranda MS, Benzi MR and Chagas BS. Determination of degree of conversion as a function of depth of a photoinitiated dental restoration composite-II application to commercial SureFil ${ }^{\mathrm{TM}}$. Polymer Testing. (Essex) 2005; 24(7):942-946.

34. Mendes LC, Tedesco AD, Miranda MS, Benzi MR and Chagas BS. Determination of degree of conversion as a function of depth of a photoinitiated dental restoration composite-III application to commercial Prodigy Condensable ${ }^{\mathrm{TM}}$. Polymer Testing (Essex) 2005; 24(8):963-968.

35. Paraizo MA, Mendes LC, Gomes AS, Miranda MS and Amaral ACF. Degradation of a dental filling material after high caries challenge. Materials Research (São Carlos) 2004; 7(4):639-642. 